\title{
Modular Subgroups, Forms, Curves and Surfaces
}

\author{
Abdellah Sebbar
}

Abstract. We study a class of subgroups of $\mathrm{PSL}_{2}(\mathbb{Z})$ which can be characterized in different ways, such as congruence groups, modular forms, modular curves, elliptic surfaces, lattices and graphs.

\section{Introduction}

In this note we study the properties of a family of subgroups of the modular group $\mathrm{PSL}_{2}(\mathbb{Z})$ which can be characterized by algebraic, number-theoretic and geometric properties, in connection with modular forms, modular curves, elliptic surfaces as well as lattices and graphs. The objects in question are the subgroups of index 12 in $\mathrm{PSL}_{2}(\mathbb{Z})$ which are genus zero and have no elliptic elements. It turns out that all these subgroups are congruence. We study the modular curves obtained from them, the elliptic surfaces having these modular curves as base curves, their graded rings of modular forms, some lattices that appear by applying the Schwarz derivative to their Hauptmoduln and their coset graphs. All these aspects are connected and most of the ideas can be generalized to subgroups of higher index.

\section{The Groups}

Let $\mathrm{PSL}_{2}(\mathbb{Z})$ be the modular group which consists of the transformations

$$
z \rightarrow \frac{a z+b}{c z+d} \quad \text { with } a, b, c, d \in \mathbb{Z}, a d-b c=1 .
$$

For convenience, the modular group and its subgroups will be represented by matrices with the understanding that a matrix and its negative will be identified. The principal congruence subgroup of level $m, m$ being a positive integer, is defined by

$$
\Gamma(m)=\left\{A \in \mathrm{SL}_{2}(\mathbb{Z}), A \equiv \pm I \bmod m\right\} /\{ \pm I\} .
$$

A congruence subgroup of level $m$ of the modular group is a subgroup which contains $\Gamma(m)$ for some positive integer $m$ and does not contain any $\Gamma(n)$ for $n<m$. Examples of such groups are the groups of unipotent and upper triangular matrices $\bmod m$ :

$$
\begin{gathered}
\Gamma_{1}(m)=\left\{A \in \mathrm{SL}_{2}(\mathbb{Z}), A \equiv \pm\left(\begin{array}{ll}
1 & b \\
0 & 1
\end{array}\right) \bmod m\right\} /\{ \pm I\}, \\
\Gamma_{0}(m)=\left\{\left(\begin{array}{ll}
a & b \\
c & d
\end{array}\right) \in \mathrm{PSL}_{2}(\mathbb{Z}), c \equiv 0 \bmod m\right\} /\{ \pm I\} .
\end{gathered}
$$

Received by the editors October $25,2000$.

AMS subject classification: $20 \mathrm{H} 05$.

(C) Canadian Mathematical Society 2002. 
It is shown in [15] that there are 33 conjugacy classes of genus zero and torsion-free congruence subgroups of the modular groups $\mathrm{PSL}_{2}(\mathbb{Z})$, see also [10] for the complete list. The indices which occur are 6, 12, 24, 36, 48 and 60. These groups share many arithmetic and geometric properties. However, the index 12 subgroups seem to be very special, and they are the subject of this note. In this regard, we have

Proposition 2.1 Up to modular conjugacies, there are six genus zero and torsion-free congruence subgroups of index 12 in $\mathrm{PSL}_{2}(\mathbb{Z})$. Namely, $\Gamma(3), \Gamma_{0}(4) \cap \Gamma(2), \Gamma_{1}(5)$, $\Gamma_{0}(6), \Gamma_{0}(8)$ and $\Gamma_{0}(9)$.

Each of them has four classes of parabolic subgroups, and each of them is canonically generated by a set of four parabolic elements. The Riemann-Hurwitz formula shows that a fundamental domain for the action of each of these groups has a hyperbolic area equal to $4 \pi$. The group $\Gamma(3)$ is conjugate to $\Gamma_{0}(9)$ by $\tau \rightarrow 3 \tau$, and the group $\Gamma_{0}(4) \cap \Gamma(2)$ is conjugate to $\Gamma_{0}(8)$ by $\tau \rightarrow 2 \tau$. Both conjugations are nonmodular. The Proof of the above proposition follows from the the above mentioned classification of the torsion-free genus zero congruence subgroups, which is based on simple number-theoretic arguments and on the study of cusp widths in the congruence case. It is interesting to know whether there are other subgroups with the same properties but without the congruence condition.

For a subgroup $\Gamma$ of finite index in the modular group $\operatorname{PSL}_{2}(\mathbb{Z})$, the stabilizer of a cusp in $\Gamma$ is a subgroup of finite index in the stabilizer of this cusp in $\mathrm{PSL}_{2}(\mathbb{Z})$. This index is referred to as the cusp width in $\Gamma$. It is also the smallest positive integer $n$ such that a modular conjugate of the transformation $\tau \rightarrow \tau+n$ is in $\Gamma$ and fixes the cusp. If we let $n_{i}, 1 \leq i \leq h$, be the cusp widths for the $h$ inequivalent cusps, then the index $\mu$ of $\Gamma$ in $\mathrm{PSL}_{2}(\mathbb{Z})$ satisfies

$$
\mu=\sum_{i=1}^{h} n_{i} .
$$

For the groups in Proposition 2.1, the cusp widths are given in Table 1. It remains to show whether any other partition of 12 into 4 positive integers provides cusp widths of some subgroup (which must then be noncongruence). Let us go back to a more general subgroup of $\mathrm{PSL}_{2}(\mathbb{Z})$ not necessarily torsion-free and not necessarily of genus zero. Let $\mu$ be the index, $h$ the number of inequivalent cusps, $e_{2}$ the number of inequivalent elliptic fixed point of order 2 , and $e_{3}$ the number of inequivalent elliptic fixed point of order 3. The Riemann-Hurwitz formula gives the genus as

$$
g=1+\frac{1}{2}\left(\frac{\mu}{6}-h-\frac{e_{2}}{2}-\frac{2 e_{3}}{3}\right) .
$$

The quadruple $\left(g, h, e_{2}, e_{3}\right)$ is called the signature of the subgroup. Conversely, for any integers $\mu>0, h>0, g \geq 0, e_{2} \geq 0$ and $e_{3} \geq 0$ which satisfy (2), there is a subgroup of $\mathrm{PSL}_{2}(\mathbb{Z})$ with signature $\left(g, h, e_{2}, e_{3}\right)$ and index $\mu$ [11]. In fact, there is a more precise statement involving the cusp widths. Let $X$ be a set of $\mu$ letters and $t$ a fixed element of $X$. We consider pairings $(x, y)$ of permutations $x$ and $y$ acting on 


\begin{tabular}{|c|c|}
\hline$\Gamma(3)$ & $3-3-3-3$ \\
\hline$\Gamma_{0}(4) \cap \Gamma(2)$ & $4-4-2-2$ \\
\hline$\Gamma_{1}(5)$ & $5-5-1-1$ \\
\hline$\Gamma_{0}(6)$ & $6-3-2-1$ \\
\hline$\Gamma_{0}(8)$ & $8-2-1-1$ \\
\hline$\Gamma_{0}(9)$ & $9-1-1-1$ \\
\hline
\end{tabular}

Table 1

$X$ satisfying $x^{2}=y^{3}=1$ and such that the group generated by $x$ and $y$ is transitive on $X$. We define the equivalence classes $(x, y)_{t}$ modulo a conjugation of $x$ and $y$ by a permutation in $\Xi_{\mu}$ fixing $t$. Then there is a one-to-one correspondence between subgroups of finite index $\mu$ in $\mathrm{PSL}_{2}(\mathbb{Z})$ and equivalence classes $(x, y)_{t}$ acting on a set $X$ of $\mu$ letters. Moreover, the subgroup has index $\mu$, a signature $\left(g, h, e_{2}, e_{3}\right)$ and cusp widths $n_{1}, n_{2} \ldots, n_{h}$ if and only if $x$ fixes $e_{2}$ letters of $X, y$ fixes $e_{3}$ letters of $X$ and $x y$ consists of $h$ disjoint cycles of lengths $n_{i}(1 \leq i \leq h)$ [11]. If we ignore the point $t$, then the correspondence is one to one between conjugacy classes of subgroups of index $\mu$ in $\operatorname{PSL}_{2}(\mathbb{Z})$ and equivalence classes $(x, y)$ modulo any permutation in $\mathfrak{S}_{\mu}$.

Going back to our situation of torsion-free genus zero subgroups of index 12 in $\mathrm{PSL}_{2}(\mathbb{Z})$, we are looking for $x$ and $y$ acting with no fixed point on a set of 4 elements with the above properties and such that $x y$ decomposes into 4 cycles of lengths $n_{1}, n_{2}$, $n_{3}, n_{4}$ with $\sum n_{i}=12$. It is not difficult (modulo some easy computer work) to check that the only partitions of 12 into 4 positive integers which are realized are those listed in Table 1, see also the last section for a simple check using graphs. The corresponding subgroups are then modular conjugate to the groups in Proposition 2.1 and thus are congruence since the principal congruence subgroups are normal in $\mathrm{PSL}_{2}(\mathbb{Z})$. We then have

Theorem 2.2 All the torsion-free genus zero subgroups of $\mathrm{PSL}_{2}(\mathbb{Z})$ of index 12 are congruence subgroups, and thus are given by Proposition 2.1

To complete the description of these groups we give the generators of the transitive group acting on the set of cosets for each subgroups. The modular group $\mathrm{PSL}_{2}(\mathbb{Z})$ is generated by the transformations $S$ and $T$ defined by

$$
S \tau=\tau+1, \quad T \tau=-1 / \tau .
$$

It is also the free product of the cyclic group $\langle T\rangle$ of order 2 and the cyclic group $\langle T S\rangle$ of order 3. Let $x$ and $y$ denotes the permutations induced by the respective actions of $T$ and TS on the cosets of the subgroups in Proposition 2.1, then the permutation decompositions for $x, y$ and $x y$ are given in Table 2. Notice that the total number of disjoint cycles in the permutations, $x, y$, and $x y$ is $\mu+2$, determining that the genus of the modular subgroup is zero, while the fact that $x$ and $y$ have no fixed points implies that the subgroup is torsion-free. 


\begin{tabular}{|c|rl|}
\hline & $x:$ & $(1,12)(2,4)(3,7)(5,11)(6,8)(9,10)$ \\
$y(3)$ & $y:$ & $(1,2,3)(4,5,6)(7,8,9)(10,11,12)$ \\
& $x y:$ & $(1,10,7)(2,5,12)(3,8,4)(6,9,11)$ \\
\hline \multirow{5}{*}{$\Gamma_{0}(4) \cap \Gamma(2)$} & $x:$ & $(1,12)(2,4)(3,6)(5,7)(8,11)(9,10)$ \\
& $y:$ & $(1,2,3)(4,5,6)(7,8,9)(10,11,12)$ \\
& $x y:$ & $(1,10,7,6)(2,5,8,12)(3,4)(9,11)$ \\
\hline & $x:$ & $(1,2)(3,4)(5,7)(6,8)(9,10)(11,12)$ \\
$\Gamma_{1}(5)$ & $y:$ & $(1,2,3)(4,5,6)(7,8,9)(10,11,12)$ \\
& $x y:$ & $(1,3,5,8,4)(6,9,11,10,7)$ \\
\hline & $x:$ & $(1,2)(3,4)(5,7)(6,10)(8,12)(9,11)$ \\
& $y:$ & $(1,2,3)(4,5,6)(7,8,9)(10,11,12)$ \\
$\Gamma_{0}(6)$ & $x y:$ & $(1,3,5,8,10,4)(6,11,7)(9,12)$ \\
& $x:$ & $(1,2)(3,4)(5,7)(6,9)(8,10)(11,12)$ \\
& $y:$ & $(1,2,3)(4,5,6)(7,8,9)(10,11,12)$ \\
& $x y:$ & $(1,3,5,8,11,10,9,4)(6,7)$ \\
\hline$\Gamma_{0}(8)$ & $x:$ & $(1,2)(3,4)(5,7)(6,10)(8,9)(11,12)$ \\
& $y:$ & $(1,2,3)(4,5,6)(7,8,9)(10,11,12)$ \\
& $x y:$ & $(1,3,5,8,7,6,11,10,4)$ \\
\hline & & \\
\hline & &
\end{tabular}

Table 2

\section{The Curves}

In this section we look at the quotients $G \backslash \mathfrak{H}$ where $G$ is one of the groups of Proposition 2.1. These quotients when compactified (by adjoining the cusps) become compact Riemann surfaces. Each of these groups has a Hauptmodul which generates the function field of these surfaces. Since the groups are also torsion-free, these Hauptmoduls provide (conformal) isomorphisms between $G \backslash \mathfrak{H}$ and the projective line $\mathbb{P}^{1}$ minus certain 4 points. If we fix three points to be 0,1 and $\infty$ (which can be done by applying a linear fractional transformation), we may ask for which complex number $z$, the curve $\mathbb{P}^{1} \backslash\{0,1, \infty, z\}$ is a modular curve, that is a quotient of the upper half-plane by a modular subgroup. Proposition 2.1 allows us to determine all the arithmetic curves of this form once we know the Hauptmoduln for the groups, and Theorem 2.2 tells us that in this way we have determined all the modular curves of the form $\mathbb{P}^{1} \backslash\{0,1, \infty, z\}$. In Table 3 we list Hauptmoduln for the six groups, normalized to have the Fourier expansion $1 / q+O(q)$ where $q$ is a uniformizer at $\infty$, in terms of the Dedekind eta function

$$
\eta(\tau)=q^{\frac{1}{24}} \prod_{n=1}^{\infty}\left(1-q^{n}\right), \quad q=\exp (2 \pi i \tau)
$$


with exception of $\Gamma_{1}(5)$. These data can be found in [7], see also [9]. Table 3 also contains the values of the Hauptmoduln at the cusps which are the locations of the punctures on $\mathbb{P}^{1}$. These values are explicit or are roots of given quadratic polynomials; they are ordered according to decreasing cusp width as given in Table 1 .

The quadratic polynomials indicate that their roots are values for the Hauptmoduln, and the order in which these values are listed follows the ordering of the cusp widths given in Table 1.

\begin{tabular}{|c|c|c|}
\hline Group & Hauptmodul & Values at the cusps \\
\hline$\Gamma(3)$ & $\left(\frac{\eta(\tau / 3)}{\eta(3 \tau)}\right)^{3}$ & $3, z^{2}+3 z+9, \infty$ \\
\hline$\Gamma_{0}(4) \cap \Gamma(2)$ & $\frac{\eta(2 \tau)^{12}}{\eta(\tau)^{4} \eta(4 \tau)^{8}}$ & $4,-4,0, \infty$ \\
\hline$\Gamma_{1}(5)$ & $\frac{1}{q} \prod_{n=1}^{\infty}\left(1-q^{n}\right)^{-5\left(\frac{n}{5}\right)}$ & $z^{2}-11 z-1,0, \infty$ \\
\hline$\Gamma_{0}(6)$ & $\frac{\eta(\tau)^{5} \eta(3 \tau)}{\eta(2 \tau) \eta(6 \tau)^{5}}$ & $5,-4,-3, \infty$ \\
\hline$\Gamma_{0}(8)$ & $\frac{\eta(4 \tau)^{12}}{\eta(2 \tau)^{4} \eta(8 \tau)^{8}}$ & $4,-4,0, \infty$ \\
\hline$\Gamma_{0}(9)$ & $\left(\frac{\eta(\tau)}{\eta(9 \tau)}\right)^{3}$ & $3, z^{2}+3 z+9, \infty$ \\
\hline
\end{tabular}

Table 3

In the expression of the Hauptmodul for $\Gamma_{1}(5),(\vdots)$ denotes the Legendre symbol. This Hauptmodul is the fifth power of the one for $\Gamma(5)$, while the values at the cusps are obtained by disymmetrizing $\Gamma_{0}(5)$. In fact if $f$ is the normalized Hauptmodul for $\Gamma_{1}(5)$ and $g$ is the normalized Hauptmodul for $\Gamma_{0}(5)$, then we have

$$
g=f-\frac{1}{f+5} .
$$

The group $\Gamma_{0}(5)$ has two cusps at which the values of $g$ are 6 and $\infty$ leading to the values for $\Gamma_{1}(5)$ in Table 3 (For simplicity, it is $f+5$ which appears in the table).

Applying a linear fractional transformation to the 4 values for each group so that each triple is sent to $0,1, \infty$, we obtain 17 values for the fourth cusp. Denoting $\exp (2 \pi i / 3)$ by $\omega$ and the roots of $z^{2}-125 z+125$ by $\alpha$ and $\beta$, we have

Theorem 3.1 The curve $\mathbb{P}^{1} \backslash\{0,1, \infty, z\}$ is a modular curve if and only if $z$ or $1 / z$ is a member of

$$
\left\{-8,-1, \frac{9}{8}, 2,9,-\omega, \alpha, \beta,-\frac{\alpha}{\beta}\right\} \text {. }
$$


So far we have considered only the quotients of the upper half-plane by subgroups of the modular group. Let us consider a more general setting in which the groups are general Fuchsian groups. We consider such group $\Gamma$ which is genus zero, has no elliptic elements and has 4 cusps (thus the index 12 condition above is translated into fundamental area being $4 \pi$ ). Moreover, we require the group to have the same set of cusps as the modular group, namely $\mathbb{O} \cup\{\infty\}$. The last condition can be assured if we assume, as we do, that the group is commensurable with the modular group.

Let $\Gamma$ be a Fuchsian group commensurable with the modular group. Using Helling's theorem this group is conjugate to a subgroup of $\Gamma_{0}(f)+$ for some squarefree positive integer $f$. In this case, every element of $\Gamma_{0}(f)+$ has the form $[3,4]$

$$
A=\left(\begin{array}{cc}
a \sqrt{e} & b / \sqrt{e} \\
c f / \sqrt{e} & d \sqrt{e}
\end{array}\right), \quad \text { with } \operatorname{det}(A)=1 \text { and } e \mid f, e>0,
$$

with $a, b, c, d, e$ being integers. If an element of this form is parabolic, then its trace, $(a+d) \sqrt{e}$, must be equal to \pm 2 . It follows that $e=1$, and the element is then in $\mathrm{PSL}_{2}(\mathbb{Z})$. If $\Gamma$ is genus zero and has no elliptic elements then so is its conjugate in $\Gamma_{0}(f)+$ which then has a set of parabolic generators. Therefore, we have

Theorem 3.2 Every torsion-free genus zero Fuchsian group commensurable with the modular group is conjugate to a subgroup of the modular group.

It follows that if we add the condition that the hyperbolic area is $4 \pi$, then the conjugate inside $\mathrm{PSL}_{2}(\mathbb{Z})$ has index 12, and thus is conjugate to one of the groups in Proposition 2.1 which can be reduced to just 4 groups under nonmodular conjugacies.

Corollary 3.3 Every torsion-free genus zero Fuchsian group commensurable with the modular group having hyperbolic area $4 \pi$ is either conjugate to $\Gamma(3), \Gamma_{1}(5), \Gamma_{0}(6)$ or $\Gamma_{0}(8)$.

Corollary 3.4 The only values of $z$ for which $\mathbb{P}^{1} \backslash\{0,1, \infty, z\}$ is a quotient of the upper half-plane by a Fuchsian group commensurable with the modular group are those given by Theorem 3.1.

\section{The Forms}

Each of the 6 groups dealt with in the previous sections has a lifting to the inhomogeneous modular group $\mathrm{SL}_{2}(\mathbb{Z})$ such that this lifting has no element of trace -2 . These groups are $\Gamma(3), \Gamma_{1}(4) \cap \Gamma(2), \Gamma_{1}(5), \Gamma_{1}(6), \Gamma_{0}(8) \cap \Gamma_{1}(4)$ and $\Gamma_{0}(9) \cap \Gamma_{1}(3)$, and they have index 24 as subgroups of $\mathrm{SL}_{2}(\mathbb{Z})$ since they do not contain $-I$. It is clear that these are the only subgroups of index 24 in $\mathrm{SL}_{2}(\mathbb{Z})$ which are of genus zero, torsionfree, and not containing $-I$. The purpose of this section is to study the properties of the spaces of modular forms for these groups. 
For a more general subgroup $\Gamma$ of $\mathrm{SL}_{2}(\mathbb{Z})$ of finite index, let $g, h, e_{2}, e_{3}$ be the data corresponding to its projective image in $\mathrm{PSL}_{2}(\mathbb{Z})$ as in Section 1 . We write $h=u+v$ where $u$ is the number of inequivalent cusps fixed by a parabolic element of trace 2 (cusps of the first kind), and $v$ is the number of inequivalent cusps fixed by a parabolic element of trace -2 (cusps of the second kind). Let $M_{n}(\Gamma)$ be the complex vector space of modular forms of integer weight $n \geq 0$ for $\Gamma$. The graded ring of modular forms with integer weights for $\Gamma$,

$$
M(\Gamma)=\bigoplus_{n=0}^{\infty} M_{n}(\Gamma)
$$

is either infinitely generated or is a polynomial ring with two generators, namely $M(\Gamma)=\mathbb{C}\left[f_{1}, f_{2}\right]$, where $f_{1}$ and $f_{2}$ are algebraically independent modular forms for $\Gamma$ with integer weights, see [1] for a nice account on these facts. In the latter case $\Gamma$ has genus zero since the degree zero part of the fraction field of the ring (which is the field of modular functions) has a single generator.

Proposition 4.1 ([1]) Up to conjugacy, there are only 17 subgroups $\Gamma$ of SL of finite index such that $M(\Gamma)=\mathbb{C}\left[f_{1}, f_{2}\right]$.

If $f_{1}$ has weight $a$ and $f_{2}$ has weight $b$, it is clear that the dimension of the complex space of modular forms of weight $n$ is equal to the coefficient of $t^{n}$ in the Taylor expansion of

$$
\frac{1}{\left(1-t^{a}\right)\left(1-t^{b}\right)} \text {. }
$$

Meanwhile, such dimensions are computed independently using the data from the group signatures, see [16]. This observation makes restrictions on the weights $a$ and $b$ and on the signatures, and leads to the above proposition.

We explain the particular case when $a=b=1$ in the above proposition: in this case, the dimension of the space of weight $n \geq 0$ modular forms for $\Gamma, M_{n}(\Gamma)$, is $n+1$. This follows from $1 /(t+1)^{2}=\sum_{n>0}(n+1) t^{n}$. From [16], these dimensions are given as follows: If $n=2$, then $\operatorname{dim} M_{n}(\Gamma)$ is equal to $g$ if $h=0$ and to $g+h-1$ if $h>0$. If $n>2$ is even, then

$$
\operatorname{dim} M_{n}(\Gamma)=(n-1)(g-1)+e_{2}\left[\frac{n}{4}\right]+e_{3}\left[\frac{n}{3}\right]+\frac{n}{2} h .
$$

If $n \geq 3$ is odd, then

$$
\operatorname{dim} M_{n}(\Gamma)=(n-1)(g-1)+e_{2}\left[\frac{n}{4}\right]+e_{3}\left[\frac{n}{3}\right]+\frac{n}{2} u+\frac{n-1}{2} v
$$

Although not used here, the situation is more complicated when $n=1$ but simple formulas for the case $\Gamma=\Gamma(m)$ or $\Gamma_{0}(m)$ can be found in the literature $[14,16]$. Since in each case, the expression equals $n+1$ for each $n$, we have, for $n=2$, that $g+h-1=3$ since $h>0$ as the groups are of finite index in $\mathrm{SL}_{2}(\mathbb{Z})$, and for $n=4$ we have $3(g-1)+e_{2}+e_{3}+2 h=5$. These relations yield $g+e_{2}+e_{3}=0$. Since $g, e_{2}$, 
$e_{3}$ are all $\geq 0$, we must have $g=e_{2}=e_{3}=0$, and then $h=4$. Using the case $n=3$, we find that $u=h=4$ and thus $v=0$. Since no parabolic element has a trace equal to -2 , then $-I$ is not in $\Gamma$. From (2) the index of the projection $\Gamma$ in $\operatorname{PSL}_{2}(\mathbb{Z})$ is 12 , and thus the index of $\Gamma$ in $\mathrm{SL}_{2}(\mathbb{Z})$ is 24 . We deduce the following result which is also stated in [1] (without the explicit form of all the groups)

Theorem 4.2 There are exactly six subgroups of $\mathrm{SL}_{2}(\mathbb{Z})$ whose graded ring of modular forms $M(\Gamma)$ is a polynomial ring with two generators each of degree 1 . Namely, $\Gamma(3)$, $\Gamma_{1}(4) \cap \Gamma(2), \Gamma_{1}(5), \Gamma_{1}(6), \Gamma_{0}(8) \cap \Gamma_{1}(4)$ and $\Gamma_{0}(9) \cap \Gamma_{1}(3)$ whose projections in $\mathrm{PSL}_{2}(\mathbb{Z})$ are given by Proposition 2.1

To complete the description of the above graded rings, and for later purposes, we provide the generators for each case. Expressions for the modular forms for $\Gamma_{1}(5)$ were kindly provided to me by Professor Masao Koike. The others can be found using the properties of Jacobi theta functions

$$
\theta_{2}(\tau)=\sum_{-\infty}^{\infty} e^{\left(n+\frac{1}{2}\right)^{2} \pi i \tau}, \quad \theta_{3}(\tau)=\sum_{-\infty}^{\infty} e^{n^{2} \pi i \tau}, \quad \theta_{4}(\tau)=\sum_{-\infty}^{\infty}(-1)^{n} e^{n^{2} \pi i \tau},
$$

which satisfy the following transformations:

$$
\begin{gathered}
\theta_{2}(\tau+1)=e^{\frac{\pi i}{4}} \theta_{2}(\tau), \quad \theta_{3}(\tau+1)=\theta_{4}(\tau), \quad \theta_{4}(\tau+1)=\theta_{3}(\tau) . \\
\theta_{2}\left(\frac{-1}{\tau}\right)=(-i \tau)^{\frac{1}{2}} \theta_{4}(\tau), \quad \theta_{3}\left(\frac{-1}{\tau}\right)=(-i \tau)^{\frac{1}{2}} \theta_{3}(\tau), \quad \theta_{4}\left(\frac{-1}{\tau}\right)=(-i \tau)^{\frac{1}{2}} \theta_{2}(\tau) .
\end{gathered}
$$

Let $\phi$ be defined by

$$
\phi(\tau)=\theta_{2}(2 \tau) \theta_{2}(6 \tau)+\theta_{3}(2 \tau) \theta_{3}(6 \tau) .
$$

Then $\phi$ is a modular form of weight 1 for $\Gamma_{1}(3)$, in particular it is a modular form for $\Gamma(3)$ and $\Gamma_{1}(6)$. Also define

$$
w=\prod_{n=1}^{\infty}\left(1-q^{n}\right)
$$

and

$$
\psi_{0}=w^{-3 / 5} \sum_{n=-\infty}^{\infty}(-1)^{n} q^{\left(5 n^{2}-n\right) / 2}, \quad \psi_{1}=w^{-3 / 5} q^{1 / 5} \sum_{n=-\infty}^{\infty}(-1)^{n} q^{\left(5 n^{2}-3 n\right) / 2} .
$$

These are forms of weight $1 / 5$ for $\Gamma(5)$ and a multiplier system, [1]. The quotient $\psi_{1} / \psi_{0}$ is a Hauptmodul for $\Gamma(5)$ and in fact we have

$$
\psi_{1} / \psi_{0}=q^{1 / 5} \prod_{n=1}^{\infty}\left(1-q^{n}\right)^{\left(\frac{n}{5}\right)},
$$

a product that occurs to the inverse fifth power in Table 3. Table 4 gives the generators $f_{1}, f_{2}$ for the graded rings; they are chosen so that $f_{1} / f_{2}$ coincide exactly with the Hauptmoduln given in the second column of Table 3. It is not difficult to see (from the $q$-expansions) that $f_{1}$ and $f_{2}$ are, in each case, linearly independent, and since they have the same weight, they are also algebraically independent. 


\begin{tabular}{|c|c|c|}
\hline Group & $f_{1}$ & $f_{2}$ \\
\hline$\Gamma(3)$ & $\phi(\tau)$ & $\frac{1}{6}(\phi(\tau / 3)-\phi(\tau))$ \\
\hline$\Gamma_{1}(4) \cap \Gamma(2)$ & $4\left(\theta_{3}(\tau)^{2}+\theta_{4}(\tau)^{2}\right)$ & $\theta_{3}(\tau)^{2}-\theta_{4}(\tau)^{2}$ \\
\hline$\Gamma_{1}(5)$ & $\psi_{0}^{5}$ & $\psi_{1}^{5}$ \\
\hline$\Gamma_{1}(6)$ & $7 \phi(2 \tau)-\phi(\tau)$ & $\phi(\tau)-\phi(2 \tau)$ \\
\hline$\Gamma_{0}(8) \cap \Gamma_{1}(4)$ & $4\left(\theta_{3}(2 \tau)^{2}+\theta_{4}(2 \tau)^{2}\right)$ & $\theta_{3}(2 \tau)^{2}-\theta_{4}(2 \tau)^{2}$ \\
\hline$\Gamma_{0}(9) \cap \Gamma_{1}(3)$ & $\phi(3 \tau)$ & $\frac{1}{6}(\phi(\tau)-\phi(3 \tau))$ \\
\hline
\end{tabular}

Table 4

\section{The Lattices}

Let us define the Schwarz derivative for a function defined on $\mathfrak{G}$ by

$$
\{f, \tau\}:=2 \frac{f^{\prime \prime \prime}}{f^{\prime}}-3\left(\frac{f^{\prime \prime}}{f^{\prime}}\right)^{2} .
$$

If $f$ is a Hauptmodul for a genus zero subgroup $\Gamma$ of $\operatorname{PSL}_{2}(\mathbb{Z})$, then $\{f, \tau\}$ is a weight 4 modular form for the normalizer of $\Gamma$ in $\operatorname{PSL}_{2}(\mathbb{R})$ which is holomorphic everywhere (including at the cusps) precisely when $\Gamma$ is torsion-free [9]. Moreover, $\{f, \tau\}$ does not depend on the choice of the Hauptmodul. Let us examine the situation for the groups under consideration using the Hauptmoduln given in Table 3. Since $\Gamma_{0}(9)$ is conjugate to $\Gamma(3)$ by $\tau \rightarrow 3 \tau$ and $\Gamma_{0}(4) \cap \Gamma(2)$ is conjugate to $\Gamma_{0}(8)$ by $\tau \rightarrow 2 \tau$, we only need to look at the case of four groups whose normalizers are given in Table 5.

The spaces of weight 4 modular forms for the normalizers all have dimension 1 except for $\Gamma_{0}(6)+$ which has dimension 2. As it was mentioned above, the Schwarz derivatives are holomorphic including at the cusps and a local analysis at $\infty$ shows that the constant coefficient of their $q$-expansion is always $4 \pi^{2} / d^{2}$ where $d$ is the cusp width at $\infty$ and the coefficient of $q^{1 / d}$ is 0 . This will allow us to completely determine these weight 4 forms once we have bases for the above spaces of weight 4 modular forms. Also these forms are expressed in terms of $f_{1}$ and $f_{2}$ of Table 4 by means of homogeneous polynomials of degree 4 . The normalizer of $\Gamma(3)$ is $\mathrm{PSL}_{2}(\mathbb{Z})$, whose space of weight 4 modular forms is 1-dimensional generated by the Eisenstein series of weight 4

$$
E_{4}(\tau)=1+240 \sum_{n=1}^{\infty}\left(\sum_{d \mid n} d^{3}\right) q^{n}
$$

Therefore the Schwarz derivative of a Hauptmodul $f$ for $\Gamma(3)$ is given by

$$
\{f, \tau\}=\pi^{2} E_{4}(\tau)
$$




\begin{tabular}{|l|c|c|c|c|}
\hline Group & $\Gamma(3)$ & $\Gamma_{0}(4) \cap \Gamma(2)$ & $\Gamma_{1}(5)$ & $\Gamma_{0}(6)$ \\
\hline Normalizer & $\mathrm{PSL}_{2}(\mathbb{Z})$ & $\Gamma_{0}(2)+$ & $\Gamma_{0}(5)+$ & $\Gamma_{0}(6)+$ \\
\hline
\end{tabular}

Table 5

This also implies that the Schwarz derivative of a Hauptmodul for $\Gamma_{0}(9)$ is given by $4 \pi^{2} E_{4}(3 \tau)$. Notice that $E_{4}$ is the theta function of the root lattice $\mathbf{E}_{8}$, and in fact, for all the other groups, the Schwarz derivative coincides with the theta function of some rank 8 lattice as we will see.

The normalizer, $\Gamma_{0}(2)+$, of $\Gamma_{0}(4) \cap \Gamma(2)$ has a space of weight 4 modular forms generated by

$$
\frac{1}{4}\left(\theta_{3}^{4}(\tau)+\theta_{4}^{4}(\tau)\right)^{2}
$$

which coincides with $1 / \pi^{2}\{f, \tau\}$ for any Hauptmodul $f$ of $\Gamma_{0}(4) \cap \Gamma(2)$, and is also the theta function of the lattice $\mathbf{D}_{4} \oplus \mathbf{D}_{4}$ (see [5] for expressions of theta functions for some lattices).

The normalizer of $\Gamma_{1}(5)$ is $\Gamma_{0}(5)+\left(\right.$ when $n$ is square-free, the normalizer of $\Gamma_{0}(n)$ and $\Gamma_{1}(n)$ are both given by $\left.\Gamma_{0}(n)+\right)$ whose space of weight 4 modular forms is 1 dimensional. We compute the Schwarz derivative using the Hauptmodul given in Table 3. It turns out, from the $q$-expansion

$1+120 q^{2}+240 q^{3}+600 q^{4}+1440 q^{5}+2400 q^{6}+3120 q^{7}+5400 q^{8}+7200 q^{9}+O\left(q^{10}\right)$

that it coincides with the theta function of the 8-dimensional 5-modular lattice $\mathbf{Q}_{8}$ (1) with determinant 625 and minimal norm 4 (also known as the icosian or Maass lattice) [6]. This theta function is indeed a modular form for $\Gamma_{0}(5)+$.

The normalizer of $\Gamma_{0}(6)$ is $\Gamma_{0}(6)+$ whose space of weight 4 modular forms has dimension 2 and is generated by [9]

$$
(\eta(\tau) \eta(2 \tau) \eta(3 \tau) \eta(6 \tau))^{2} \quad \text { and } \quad h_{1}^{2}\left(1-4 h_{2}-16 h_{2}^{3}+16 h_{2}^{4}\right)
$$

where

$h_{1}(\tau)=\left(\theta_{3}(\tau) \theta_{3}(2 \tau) \theta_{3}(3 \tau) \theta_{3}(6 \tau)\right)^{2} \quad$ and $\quad h_{2}(\tau)=\frac{\eta(\tau / 2) \eta(3 \tau / 2) \eta(4 \tau) \eta(12 \tau)}{\eta(\tau) \eta(2 \tau) \eta(3 \tau) \eta(6 \tau)}$

Since the constant term of the Schwarz derivative of a Hauptmodul $f$ for $\Gamma_{0}(6)$ is $4 \pi^{2}$ and the coefficient of $q$ is 0 , we can see that $1 / 4 \pi^{2}\{f, \tau\}=h_{1}^{2}\left(1-4 h_{2}-16 h_{2}^{3}+16 h_{2}^{4}\right)$ which coincides with the theta function of $\mathbf{A}_{2} \otimes \mathbf{D}_{4}$, see [13].

In Table 6 we gather these lattices which thus appeared in a natural way in connection with our groups. We also give the homogeneous polynomial $P(x, y)$ of degree 4 which expresses their theta functions in terms of $f_{1}$ and $f_{2}$ as given by Table 4 . We do not know what characterizes these lattices from the other rank 8 lattices. 


\begin{tabular}{|c|c|c|}
\hline Group & $\frac{1}{4 \pi^{2}}\{f, \tau\}$ & $P(x, y)$ \\
\hline$\Gamma(3)$ & $\theta_{E_{8}}(\tau)$ & $x^{4}+216 x y^{3}$ \\
\hline$\Gamma_{1}(4) \cap \Gamma(2)$ & $\theta_{D_{4} \oplus D_{4}}(\tau)$ & $1 / 2^{12}\left(x^{2}+16 y^{2}\right)^{2}$ \\
\hline$\Gamma_{1}(5)$ & $\theta_{Q_{8}(1)}(\tau)$ & $x^{4}-12 x^{3} y+134 x^{2} y^{2}+12 x y^{3}+y^{4}$ \\
\hline$\Gamma_{1}(6)$ & $\theta_{A_{2} \otimes D_{4}}(\tau)$ & $1 / 6^{4}\left(x^{4}+4 x^{3} y+54 x^{2} y^{2}+388 x y^{3}+769 y^{4}\right)$ \\
\hline$\Gamma_{0}(8) \cap \Gamma_{1}(4)$ & $\theta_{D_{4} \oplus D_{4}}(2 \tau)$ & $1 / 2^{12}\left(x^{2}+16 y^{2}\right)^{2}$ \\
\hline$\Gamma_{0}(9) \cap \Gamma_{1}(3)$ & $\theta_{E_{8}}(3 \tau)$ & $x^{4}+216 x y^{3}$ \\
\hline
\end{tabular}

Table 6

\section{The Surfaces}

An elliptic surface is a complex surface $X$ together with a holomorphic map $\pi: X \rightarrow$ $C$, where $C$ is a smooth curve, such that the general fiber of $\pi$ is a smooth connected curve of genus 1 . We suppose that a distinguished global section $S_{0}$ exists, so that the general fibers can be seen as elliptic curves. We will always assume that there are no exceptional curves in the fibers. Kodaira has classified all the fiber types into two infinite families $\left(I_{n}, I_{n}^{*}, n \geq 0\right)$ and six exceptional cases. An elliptic surface is semistable if all the singular fibers are of type $I_{n}$, that is, the fiber is a cycle of $n$ irreducible curves. If $\Gamma$ is a torsion-free subgroup of $\mathrm{SL}_{2}(\mathbb{Z})$, following Kodaira's construction, we consider the quotient of $\mathfrak{H} \times \mathbb{C}$, where $\mathfrak{H}$ is the upper-half plane, by the semi-direct product of $\Gamma$ and $\mathbb{Z}^{2}$ acting by automorphisms of the form $(\tau, z) \mapsto$ $\left(\gamma \cdot \tau,(c \tau+d)^{-1}(z+m \tau+n)\right)$, where $\gamma=\left(\begin{array}{l}a b \\ c d\end{array}\right) \in \Gamma$ and $m, n$ are integers, see [8] p. 580. This surface extends to an elliptic surface over $\Gamma \backslash \mathfrak{H}^{*}=\Gamma \backslash \mathfrak{S} \cup\{$ cusps $\}$ such that the singular fibers are above the cusps. This construction can be made even for $\Gamma$ having elliptic elements [17]. The surface obtained (or any surface isomorphic to it as a fibration) is called an elliptic modular surface. The Mordell-Weil group (or the group of global sections) is finite. A cusp of the first kind gives a singular fiber of type $I_{n}$ where $n$ is the cusp width, while a cusp of the second kind gives a fiber of type $I_{n}^{*}$. For a semistable elliptic modular surface over a curve $C \cong \mathbb{P}_{1}$, the geometric genus of the surface is given by $p_{g}=h / 2-2$ and the arithmetic genus $p_{a}$ is equal to $p_{g}$ since the irregularity of the surface is equal to the genus of the base curve which is 0 .

The inhomogeneous subgroups of $\mathrm{SL}_{2}(\mathbb{Z})$ of Section 4 provide 6 semistable elliptic surfaces with four singular fibers; the type $I_{n}$ of each singular fiber is given by the corresponding cusp width from Table 1 . These surfaces are unique and thus determine 
uniquely the 6 groups in view of the following

Proposition 6.1 ([2]) The minimal number of singular fibers for a semistable elliptic surface over a genus zero curve is four. Moreover, there are (up to isomorphism) only six such surfaces with four singular fibers.

This says in particular that all semistable elliptic surfaces with four singular fibers over a genus zero base curve are modular.

The six groups can also be determined uniquely using elliptic surfaces from another point of view. An elliptic surface is extremal if it has a finite Mordell-Weil group (MW) and a maximal Picard number, that is, the rank of the Neron-Severi group of the surface (the group of divisors on the surface modulo algebraic equivalence) is maximal. A surface is said to be rational if it is birational to $\mathrm{P}^{2}$.

Proposition 6.2 ([12]) There are 16 possible fiber types of extremal rational elliptic surfaces of which only six are semistable.

When the elliptic surface is rational, there are several constraints on the numerical invariants of the surface, in particular the Picard number is 10 . With these constraints, it is shown in [12] that the number of singular fibers is either 2, 3 or 4 . In the case of 4 singular fibers, these fibers must be all semistable of types $I_{n_{i}}, i \in\{1,2,3,4\}$, such that $\sum n_{i}=12$ and $\prod n_{i}=|\mathrm{MW}|^{2}$, where $|\mathrm{MW}|$ denotes the order of the Mordell-Weil group MW. The only partitions of 12 into four positive integers whose product is a square are those partitions given in Table 1 and so extremal rational elliptic surfaces with the maximal number (which is 4 ) of singular fibers determine the six groups uniquely.

The generic fiber of an elliptic surface can be seen as an elliptic curve over the function field of the base curve. If the base curve is the compactified quotient of one the six torsion-free genus zero subgroups, and if $t$ is Hauptmodul for this group, the $J$-invariant of the elliptic curve will be a rational function of $t$ satisfying

$$
j(\tau)=J(t(\tau)), \quad \tau \in \mathfrak{H},
$$

where $j$ is the elliptic modular function (normalized Hauptmodul for $\operatorname{PSL}_{2}(\mathbb{Z})$ ). Since the subgroups have index 12 in $\mathrm{PSL}_{2}(\mathbb{Z})$, a Hauptmodul for $\mathrm{PSL}_{2}(\mathbb{Z})$ is a rational function of degree 12 of each subgroup Hauptmodul. Knowing the cusp widths, the values of the the normalized Hauptmoduln at the cusps and at ramification points of $j$ and the fact that the groups are torsion-free, we are able to write down the explicit rational function for each group which are listed in Table 7. The numerators are cubes as expected, and each of these rational functions is the modulus of a (local) Weierstrass equation for the corresponding elliptic surface

$$
y^{2}=x^{3}+A(t) x+B(t)
$$

where

$$
J(t)=1728 \frac{4 A(t)^{3}}{4 A(t)^{3}+27 B(t)^{2}} .
$$




\begin{tabular}{|c|c|}
\hline$\Gamma(3)$ & $\frac{\left(t^{3}\left(t^{3}+216\right)^{3}\right.}{\left(t^{3}-27\right)^{3}}$ \\
\hline$\Gamma_{0}(4) \cap \Gamma(2)$ & $\frac{\left.t^{4}+224 t^{2}+256\right)^{3}}{t^{2}(t-4)^{4}(t+4)^{4}}$ \\
\hline$\Gamma_{1}(5)$ & $\frac{\left(t^{4}+228 t^{3}+494 t^{2}+228 t+1\right)^{3}}{t\left(t^{2}-11 t-1\right)^{5}}$ \\
\hline$\Gamma_{0}(6)$ & $\frac{(t+7)^{3}\left(t^{3}+237 t^{2}+1443 t+2287\right)^{3}}{(t+3)^{2}(t+4)^{3}(t-5)^{6}}$ \\
\hline$\Gamma_{0}(8)$ & $\frac{\left(t^{4}+240 t^{3}+2144 t^{2}+3840 t+256\right)^{3}}{t(t+4)^{2}(t-4)^{8}}$ \\
\hline$\Gamma_{0}(9)$ & $\frac{(t+6)^{3}\left(t^{3}+234 t^{2}+756 t+2160\right)^{3}}{\left(t^{2}+3 t+9\right)(t-3)^{9}}$ \\
\hline
\end{tabular}

Table 7

From the global point of view, $A$ and $B$ are sections of $L^{4}$ and $L^{6}$ where $L^{-1}$ is th normal line bundle to the zero-section of the elliptic surface. In terms of the homogeneous coordinate $[u: v]$ of the base curve (which we look at as $\mathbb{P}^{1}$ for the moment), $A(u, v)$ (resp. $B(u, v)$ is a degree 4 (resp. 6) form.

Let $\Gamma$ be one the six groups. According to Section 4, the graded ring of modular forms of integer weights for $\Gamma$ is a polynomial ring $\mathbb{C}\left[f_{1}, f_{2}\right]$ where $f_{1}$ and $f_{2}$ are weight 1 modular forms. It follows that $\operatorname{Proj}\left(\mathbb{C}\left[f_{1}, f_{2}\right]\right) \cong \mathbb{P}^{1}$ where $f_{1}$ is identified with $u$ and $f_{2}$ is identified with $v$. This amounts torputting $t=u / v$ in Table 7 . Writing the numerators (which are already cubes) as $1728 \cdot 4 A(u, v)^{3}$, and the denominator as $\Delta$, we find that $\Delta-4 A(u, v)^{3}$ is a square that we write as $B(u, v)^{2}$. With a choice of sign for $B(u, v)$, and using the expressions from Table 4 , the Weierstrass equation becomes (after a standard transformation):

$$
y^{2}=4 x^{3}-g_{2} x-g_{3}
$$

where $g_{2}$ and $g_{3}$ are the classical Eisenstein series of weight 4 and 6 .

\section{The Graphs}

In Section 2, we stated that there are only six partitions of 12 into four positive integers which are the disjoint cycle length of $x y$ where $x$ and $y$ are permutations acting fixed-point freely on a set of four points such that $x^{2}=y^{3}=1$ and the group generated by $x$ and $y$ is transitive on the four points. In this section, we describe some coset graphs which determine uniquely the (conjugacy classes of) the pairings $(x, y)$.

The following five graphs are the only undirected connected trivalent graphs with four vertices. 


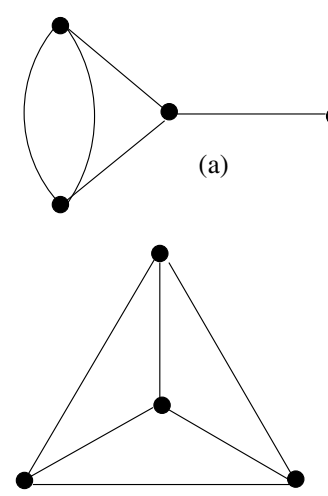

(c)

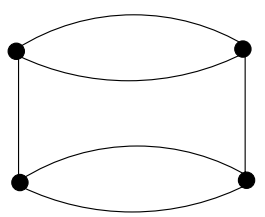

(d)

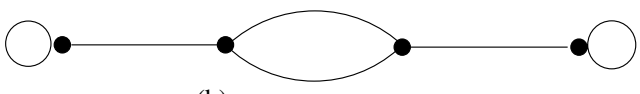

(b)

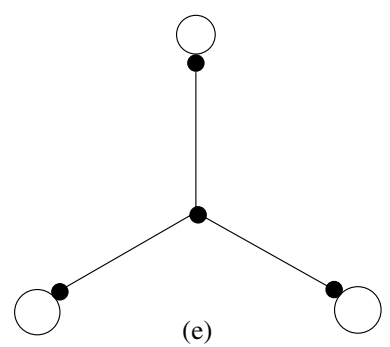

(e)

Trivalent connected 4-vertex graphs

We treat these graphs as directed graphs in which each edge is seen as a doubly directed edge and each vertex is interpreted as a positively oriented triangle:
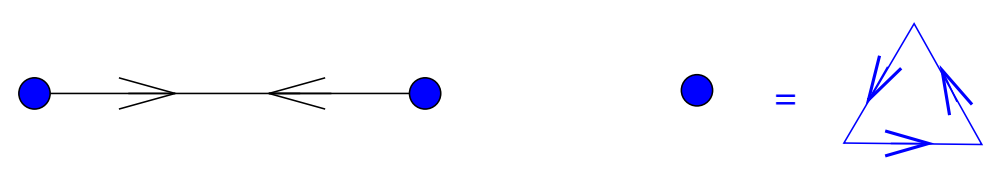

yielding directed connected trivalent graphs with 12 vertices that we still call (a), ... (e). The doubly directed edges represent an element $y$ of order 2 while the positively oriented triangles represent an element $x$ of order 3. Both $x$ and $y$ are acting fixedpoint freely on the vertices since we cannot have loops on the new graph. Now, since the graph is connected, the group generated by $x$ and $y$ is transitive on the vertices. It is clear that this process give more than five nonisomorphic graphs. To be more precise, with orientations in place, the first two undirected graphs give two more apparently different graphs, by taking the spikes outside or inside:
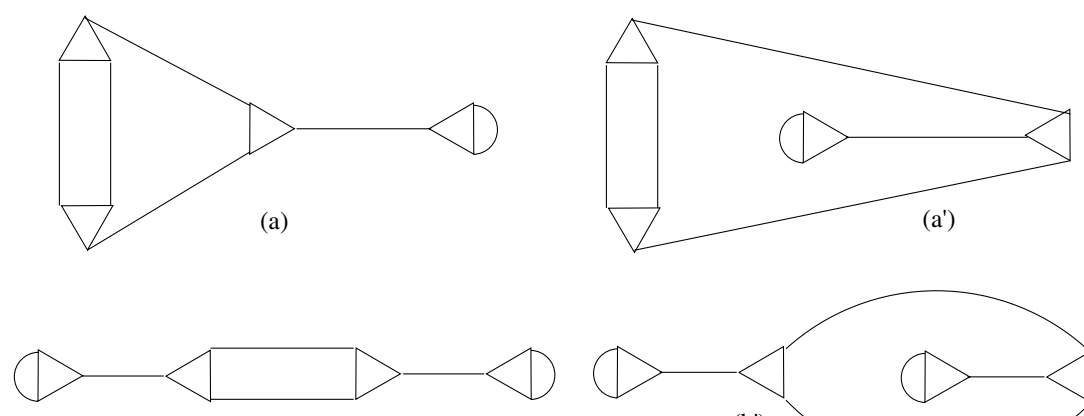

(b)

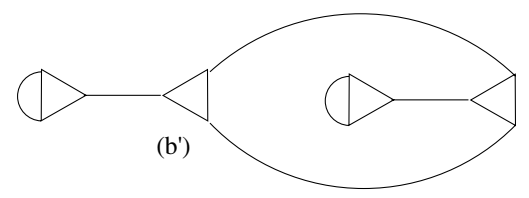

It is clear that the graphs (a) and $\left(\mathrm{a}^{\prime}\right)$ are isomorphic as directed graphs, whereas (b) and $\left(b^{\prime}\right)$ are not (they have different circuits). It is also clear that there are no other graphs of this type beside (a), (b), (c), (d), (e) and ( $\left.b^{\prime}\right)$ (this can be seen directly 
instead of shrinking the triangles to get to the trivalent graphs with four vertices). To express the cycle decomposition of the permutation $x y$, we chose a vertex and we apply $x$, then $y$, that is we take an edge (doubly oriented) followed by a side of a triangle (always in the positive orientation) until we return back to the original vertex. The length of this circuit gives the length of a cycle of $x y$. We thus obtain the permutations of Table 2 after reordering the vertices (this corresponds to conjugating $x$ and $y$ by the same permutation). We find there are only six conjugacy classes of torsion-free genus zero subgroups of index 12 in $\mathrm{PSL}_{2}(\mathbb{Z})$. At the graph level, the absence of loops correspond to the absence of elliptic elements, and the genus zero condition comes simply from the fact that we have planar connected graphs (with Euler characteristic equal to $2=2-2 g$ ).

\section{References}

[1] E. Bannai, An observation on modular forms. preprint.

[2] A. Beauville, Les familles stables de courbes elliptiques sur $\mathbb{P}_{1}{ }_{1}$ admettant quatre fibres singulières. C. R. Acad. Sci. Paris Sér. I Math. (19) 294(1982), 657-660.

[3] J. H. Conway, Understanding groups like $\Gamma_{0}(N)$. Groups, difference sets, and the Monster, Columbus, Ohio, 1993, 327-343, Ohio State Univ. Math. Res. Inst. Publ. 4, de Gruyter, Berlin, 1996.

[4] J. H. Conway and S. P. Norton, Monstrous moonshine. Bull. London Math. Soc. (3) 11(1979), 308-339.

[5] J. H. Conway and N. J. A. Sloane, Sphere Packings, Lattices and Groups. Grundlehren der Mathematischen Wissenschaften Springer-Verlag, New York, 1993.

[6] Low-Dimensional Lattices II: Subgroups of GL $(n, Z)$. Proc. Roy. Soc. London Ser. A 419(1988), 29-68.

[7] D. Ford, J. McKay and S. Norton, More on replicable functions. Comm. Algebra 22(1994), 5175-5193.

[8] K. Kodaira, On compact complex analytic surfaces II. Ann. of Math. 77(1963), 563-626.

[9] J. McKay and A. Sebbar, Fuchsian groups, automorphic functions and Schwarzians. Math. Ann. (2) 318(2000), 255-275.

[10] J-invariants of the arithmetic semistable elliptic surfaces and graphs. AMS-CRM lecture notes series, to appear.

[11] M. H. Millington, Subgroups of the classical modular group. J. London Math. Soc. (2) 1(1969), 351-357.

[12] R. Miranda and U. Persson, On extremal rational elliptic surfaces. Math. Z. (4) 193(1986), 537-558.

[13] E. M. Rains and N. J. A. Sloane, The shadow theory of modular and unimodular lattices. J. Number Theory (2) 73(1998), 359-389.

[14] Robert A. Rankin, Modular forms and functions. Cambridge University Press, 1977.

[15] A. Sebbar, Classification of genus zero torsion-free congruence groups in $\mathrm{PSL}_{2}(\mathbb{Z})$. Proceedings of the Amer. Math. Soc., to appear.

[16] G. Shimura, Introduction to the arithmetic theory of automorphic functions. Princeton University Press, Princeton, 1971.

[17] T. Shioda, On elliptic modular surfaces. J. Math. Soc. Japan 24(1972), 20-59.

Department of Mathematics and Statistics

University of Ottawa

Ottawa, Ontario

K1N 6 N5

e-mail: sebbar@mathstat.uottawa.ca 